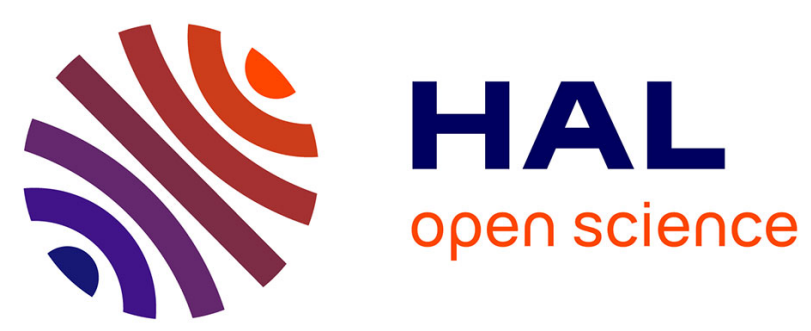

\title{
L'utilisation des helicoptères
}

Philippe Millischer

\section{- To cite this version:}

Philippe Millischer. L'utilisation des helicoptères. Revue forestière française, 1975, 27 (S), pp.466-472. 10.4267/2042/21014 . hal-03396406

\section{HAL Id: hal-03396406 https://hal.science/hal-03396406}

Submitted on 22 Oct 2021

HAL is a multi-disciplinary open access archive for the deposit and dissemination of scientific research documents, whether they are published or not. The documents may come from teaching and research institutions in France or abroad, or from public or private research centers.
L'archive ouverte pluridisciplinaire HAL, est destinée au dépôt et à la diffusion de documents scientifiques de niveau recherche, publiés ou non, émanant des établissements d'enseignement et de recherche français ou étrangers, des laboratoires publics ou privés. 


\section{L'utilisation des hélicoptères}

Depuis le début du $X X{ }^{\text {e }}$ siècle, l'aviation - ensemble des aéronefs à voilures fixes - a fait des progrès considérables dont les applications civiles ont révolutionné le monde et la vie de ses habitants.

Bien qu'imaginée à la fin du XVe siècle par le talentueux Léonard de Vinci, la giraviation — ensemble des aéronefs à voilures tournantes - devra attendre la veille de la 2 e Guerre mondiale pour devenir une réalité pratique et la Guerre de Corée pour que le plus représentatif de ses produits - l'hélicoptère - soit construit en série et employé de façon systématique.

Cet appareil aux possibilités étonnantes dont la particularité essentielle réside dans le vol vertical prend une place de plus en plus grande dans la vie moderne en remplissant de nombreuses missions civiles et militaires dans des domaines les plus divers comme par exemple :

- le travail et le transport aérien,

- la prospection pétrolière,

- l'agriculture,

- I'aide à la science,

- la protection et la sauvegarde des personnes,

- et aussi la protection des forêts.

Nous allons voir dans les lignes qui vont suivre le rôle que peut jouer l'hélicoptère dans la lutte contre les feux de forêts, les matériels actuels et en étude mis à sa disposition pour accomplir certaines missions, la place tenue actuellement par I'hélicoptère en France et à l'étranger dans la lutte contre ce fléau.

On peut savoir que les hélicoptères sont en général classés de la façon suivante :

- Hélicoptères légers - dont la charge offerte peut varier de 3 à 12 passagers ou 300 à $1200 \mathrm{~kg}$ (Alouette III, Lama, Gazelle, Dauphin pour I'Aérospatiale France; Bell 47G, Bell 206 pour les ÉtatsUnis; Bolkow 105 pour l'Allemagne).

- Hélicoptères moyens - charge offerte 13 à 35 passagers soit 1300 à 5000 kg (SA 330 Puma, SA 321 Super Frelon pour l'Aérospatiale France; Bell 212, Sikorsky S.58T, S.61 N pour les États-Unis).

- Hélicoptères lourds - charge offerte supérieure à 35 passagers et $5000 \mathrm{~kg}$ pouvant atteindre $30000 \mathrm{~kg}$ (Sikorsky S.64, CH.53 pour les États-Unis; Mi 6, Mi 8, Mi 12 pour I'U.R.S.S.).

Ils peuvent être équipés des moyens radio nécessaires aux communications avec le sol ou avec d'autres aéronefs, lesquels sont en général :

- 1 poste VHF/UHF (Very/Ultra High Frequency) réservé aux communications à courte distance et en principe de portée optique:

- 1 poste HF (High Frequency) communication à longue distance, a priori superflu dans ce genre d'opération tout au moins en Europe;

- 1 poste portatif de communication rudimentaire pour communication très courte distance. 


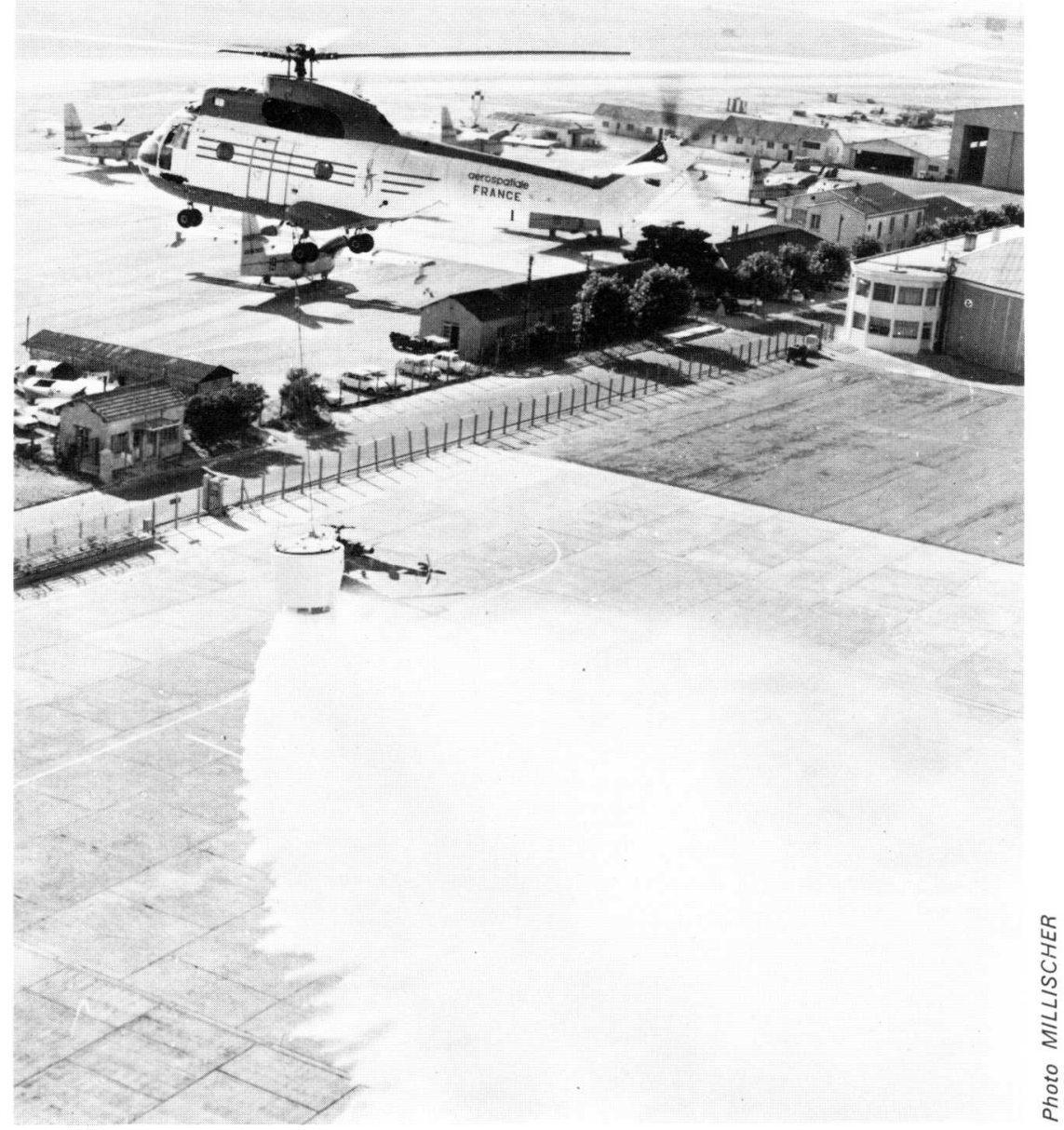

Le Puma et le seau Alkan en essai sur le parking de Marignane

\section{RÔLE DE L'HÉLICOPTÈRE}

Les possibilités quasiment universelles de I'hélicoptère peuvent lui permettre de remplir les missions les plus variées et le rendre nécessaire sinon indispensable dans le dispositif de lutte anti-incendie.

Il peut être utilisé dans les missions de prévention, d'observation et de poste de commandement volant, de transport de personnel et de matériel, d'attaque directe sur le feu d'épandage de produits chimiques et d'évacuation de blessés.

\section{MISSIONS DE PRÉVENTION, D'OBSERVATION ET DE POSTE DE COMMANDEMENT VOLANT}

Missions de prévention - peuvent être assurées par des hélicoptères légers transformés en « laboratoires volants » et qui permettent le prélèvement des végétaux dans des régions susceptibles d'incendies et le traitement immédiat des informations par le personnel spécialisé qui compose son équipage (Alouette III). 
Missions d'observation - qui sont confiées aux appareils de plus faibles capacités (Alouette II) l'équipage étant composé d'un pilote et d'un observateur participant à la détection du feu et complétant ainsi les observations des postes vigies.

Missions de poste de commandement - les moyens mis en place pour lutter contre les feux de forêts doivent être dirigés d'un poste d'observation bénéficiant d'une vue générale du théâtre des opérations.

L'hélicoptère qui a la possibilité de voler à faible vitesse et de faire du vol stationnaire est un observatoire idéal à bord duquel les spécialistes du feu et de la forêt peuvent guider avec le plus d'efficacité possible les moyens terrestres et aériens engagés dans le dispositif de lutte.

Le temps de vol nécessaire pour une telle mission ne doit pas poser de problèmes puisque l'autonomie normale de la machine (environ $3 \mathrm{~h}$ ) peut être augmentée en prévoyant un ravitaillement en carburant à proximité de la zone d'intervention dans une aire suffisamment dégagée pour permettre l'atterrissage. Les appareils légers du type Alouette III (1 pilote +6 passagers) semblent particulièrement adaptés à ce genre de missions.

\section{TRANSPORT DE PERSONNEL ET DE MATÉRIEL (HÉLITRANSPORT)}

Les spécialistes des feux de forêts conviennent que le meilleur moyen de lutte est terrestre et que l'intervention de l'homme contre les flammes est de loin plus efficace que tout autre moyen d'intervention.

Si certaines régions privilégiées disposent d'un réseau routier suffisamment équipé pour permettre l'accès des véhicules et des hommes (la Provence, le Var), il est des endroits plus difficiles d'accès comme par exemple la Corse, la Lozère, l'Ardèche et l'Hérault qui nécessitent pour une intervention rapide, des moyens aériens afin d'amener à pied d'œuvre pompiers et matériels.

Cette mission peut être assurée par des appareils légers (Alouette III, Lama) ou moyen (Puma SA 330). Sur de courtes distances I'hélicoptère peut transporter des charges externes (au crochet) dont la manipulation simple permet des rotations rapides entre le Camp de Base et la zone d'opération.
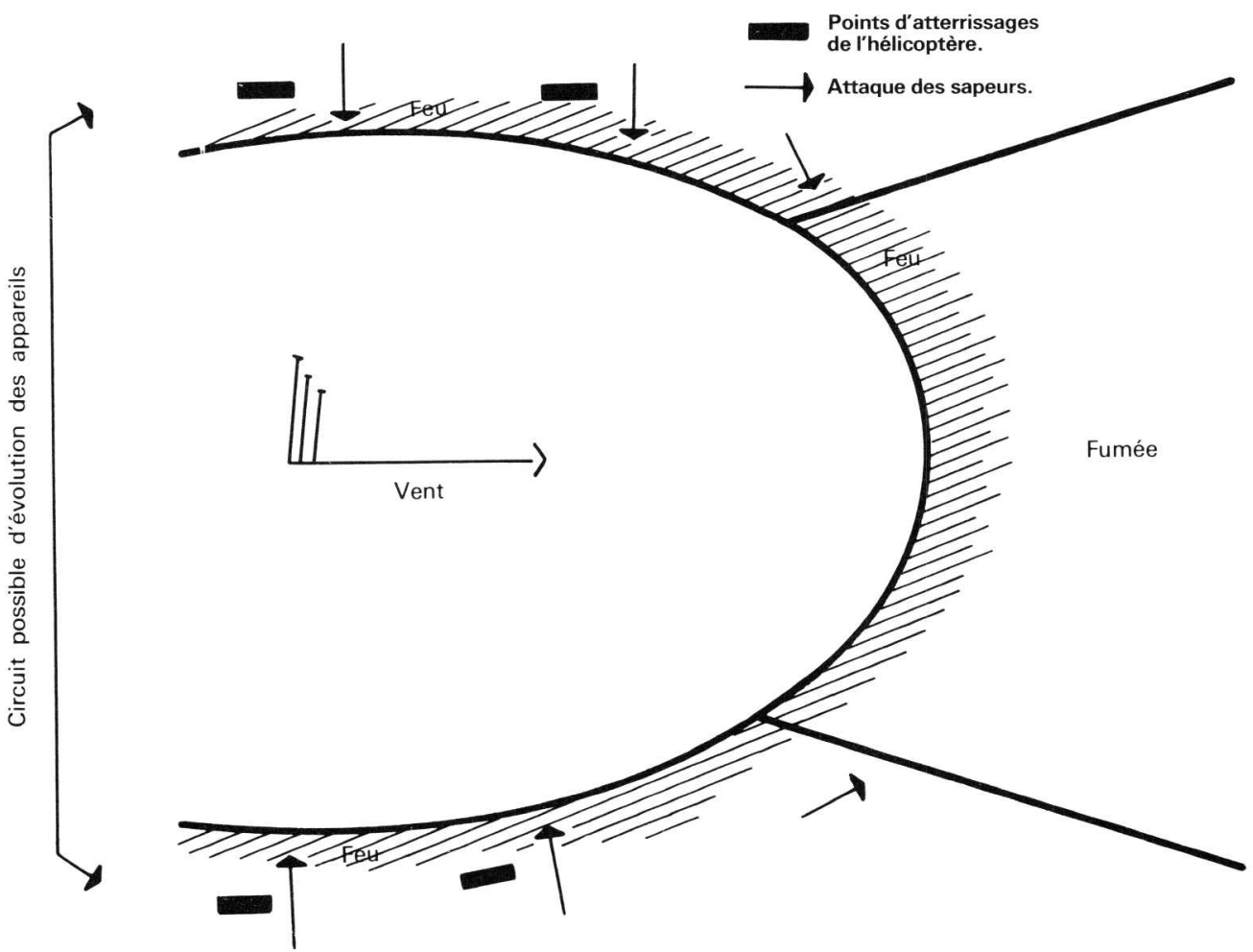
Citons par exemple, le Lama qui a une capacité maximum au crochet de $1000 \mathrm{~kg}$ et le Puma de $3000 \mathrm{~kg}$; le Super Frelon pouvant transporter jusqu'à $5000 \mathrm{~kg}$.

L'Alouette III ou le Lama pourra, par exemple, amener à pied d'œuvre une équipe de 3 hommes équipés de pompes légères munies d'un tuyau de 25 à 30 mètres et une citerne souple au crochet de 300 I d'eau ; l'appareil effectuera des rotations entre la base et le feu pour renforcer, en hommes et en matériel (eau, outils divers, scies, haches etc...), le dispositif de combat.

Le Puma pourra transporter des équipes plus importantes (20 hommes), du matériel plus lourd mais pour des raisons de manipulation sur le terrain, les citernes souples devront être limitées à une certaine contenance (800 I environ).

Ces équipes et ces matériels seront, si besoin, transportés rapidement vers d'autres zones en feu dès que leur intervention ne se fera plus nécessaire sur le terrain en cours de traitement ou lorsqu'en particulier les moyens terrestres auront atteint le sinistre (méthode actuellement appliquée en Californie par le « Department of conservation division of forestry »).

\section{AtTAque DiREcte SUR LE FEU}

L'attaque directe au feu par les moyens aériens est pour l'instant réservée aux appareils à voilures fixes de type amphibie comme le Canadair qui, par la masse d'eau larguée sur le feu (en moyenne 5000 I) provoque un effet de souffle ou de «matraque » qui permet aux équipes de pompiers au sol de s'approcher du foyer.

L'hélicoptère qui, en France, dans ce domaine n'en est qu'au stade expérimental pourrait intervenir sur le feu au moyen d'un seau au crochet à vidange rapide d'une capacité moyenne de 1600 à 2000 I et se ravitailler en eau à proximité de la zone sinistrée, dans une piscine, une retenue d'eau forestière, une rivière, un canal ou enfin dans des citernes prévues à cet effet dans le dispositif de lutte.

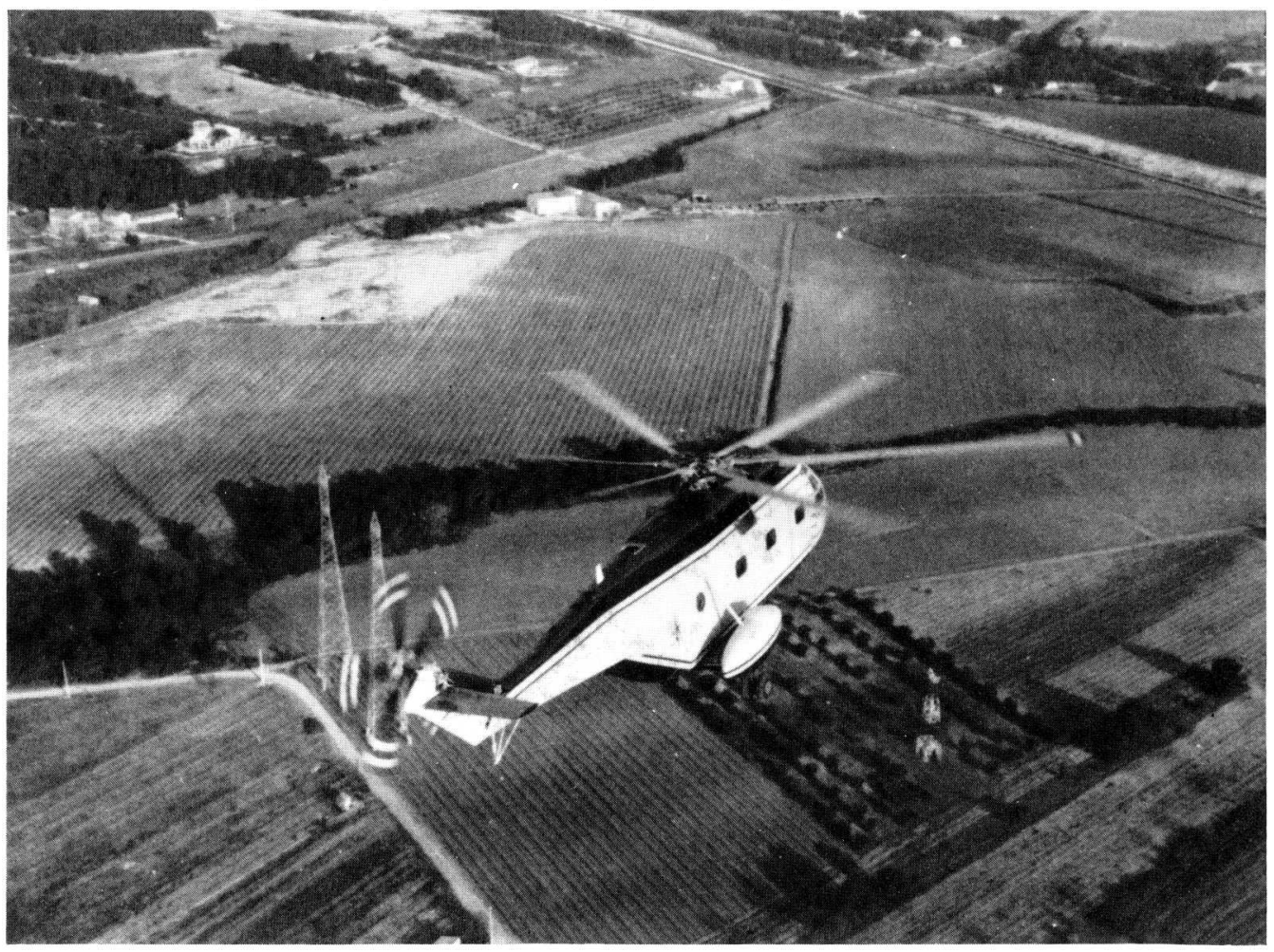


Il est démontré qu'en Provence et dans les Alpes-Maritimes I'hélicoptère peut se ravitailler en eau dans un rayon de $4 \mathrm{~km}$ de toute zone susceptible de feu, ces points de ravitaillement étant naturels ou artificiels.

La distance eau-feu joue un rôle considérable dans le rendement théorique de ce moyen de lutte. Le Puma par exemple pourra larguer au feu, pour une mission type de 2 h 45 de vol, 63 t d'eau pure en tenant compte des hypothèses opérationnelles suivantes :

- distance base d'alerte au feu : $20 \mathrm{~km}$,

- distance feu-ravitaillement en eau : $4 \mathrm{~km}$,

- capacité du seau : 1800 I,

- nombre de rotations : 35 ,

- tonnes larguées : $63 \mathrm{t}$.

Tonnes eau larguée

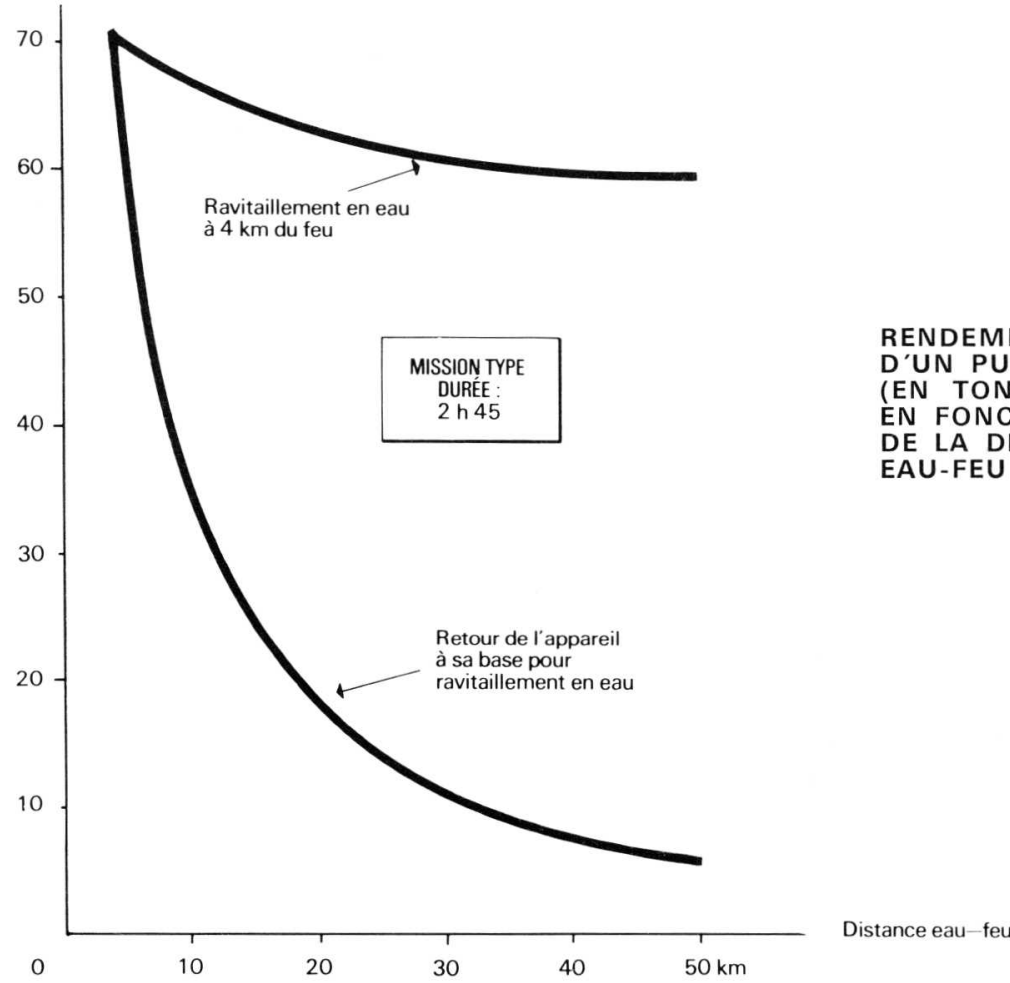

Si ce même Puma devait se ravitailler en eau à sa base de départ, soit $20 \mathrm{~km}$ le nombre de rotations tomberait à 11 et le nombre de tonnes larguées à 19, soit une baisse considérable du rendement qui condamne irrémédiablement le principe.

II est difficile d'apprécier aujourd'hui l'efficacité de l'attaque d'un ou de plusieurs Puma sur un feu violent cet appareil n'ayant jamais été utilisé de façon systématique sur les incendies de forêts, on peut dire cependant que le Puma peut empêcher l'extension latérale du feu, contribuer efficacement à la «finition » de l'incendie par des largages précis sur des foyers encore dangereux et lutter à part entière dans les feux de savanes ou de maquis.

\section{ÉPANDAGE DE PRODUITS CHIMIQUES}

Les produits chimiques sont couramment utilisés aux États-Unis par l'U.S. Forest Service qu'il s'agisse d'ignifugeant ou de gélifiant et I'hélicoptère même léger comme le Bell $47 \mathrm{G} 2$ participe activement à l'épandage de ces produits. 
L'ignifugeant (solution de borate ou de phosphate diamonique) peut être largué par l'hélicoptère au moyen d'un seau au crochet et la précision du largage (basse vitesse de translation) permet de dresser des barrières chimiques d'arrêt même dans les zones les plus inaccessibles, la taille de l'appareil étant choisie suivant l'importance de la surface à traiter.

Le gélifiant (solution d'alginate ou de carboxyméthyl) ou eau gélifiée est un retardant répandu sur le feu au moyen d'un canon à eau. Ce travail ne peut être assuré que par des hélicoptères moyens ou lourds. II convient de souligner que l'utilisation des produits chimiques n'est pas encore généralisée en France.

\section{ÉVACuATION DES BLESSÉS}

Simple rappel d'une mission universellement connue de I'hélicoptère.

\section{MATÉRIELS ACTUELS ET EN ÉTUDE}

\section{Le seau au crochet}

On peut citer dans ce domaine le seau " Alkan » expérimenté en France en 1972 par l'Aérospatiale et l'Entente interdépartementale de la préfecture des Bouches-du-Rhône et porté par l'hélicoptère Puma.

Ce seau a une contenance modulée de 1600 à 2150 litres - contenance moyenne $1800 \mathrm{I}$. L'ouverture des vannes est commandée depuis le poste de pilotage par un système électrique. Le remplissage s'accomplit en $15 \mathrm{~s}$ par immersion, et le largage de l'eau sur le feu se fait à une vitesse optimale de 30 à $40 \mathrm{~km} / \mathrm{h}$ à une hauteur la plus basse possible compte tenu des flammes, de la fumée et des turbulences thermiques.

\section{Citerne souple interne}

Elle a été expérimentée en 1967 en Provence sur l'hélicoptère lourd Mi 6 d'origine soviétique. Sa capacité est de $11 \mathrm{t}$ avec un orifice de largage rapide dont l'effet de choc n'a pas été démontré pendant les essais malgré la masse d'eau larguée.

\section{Citerne souple externe - au crochet}

D'utilisation courante dans les moyens de lutte terrestre ces citernes aérotransportables et capables de supporter des chutes verticales de quelques centaines de mètres permettent d'emmagasiner à proximité des foyers une quantité d'eau très importante. II convient, comme il a été dit plus haut de ne pas utiliser de trop grandes citernes pour des besoins de manutention au sol.

\section{Canon à eau ou lance-canon}

Ce système peut être monté sur des hélicoptères moyens ou lourds et il fut expérimenté en 1967 sur le Mi 6; mais la durée d'expérimentation n'a pas été suffisante pour en dégager tous les enseignements. II peut permettre un tir précis sur les flammes dans la mesure où le jet peut traverser le souffle du rotor principal sans dispersion ce qui semble possible si le liquide est éjecté sous une pression de l'ordre de 15 bars pour le Puma par exemple. Ce canon à eau est peut-être l'équipement futur de I'hélicoptère pour l'attaque directe du feu mais encore faut-il aller plus loin dans l'expérimentation.

\section{PLACE TENUE ACTUELLEMENT PAR L'HÉLICOPTÈre DANS LA LUTTE CONTRE LES INCENDIES DE FORÊTS}

En France l'hélicoptère est pratiquement ignoré dans le dispositif de lutte contre les feux de forêts qu'il s'agisse des appareils légers ou qu'il s'agisse des appareils moyens ou lourds.

La Protection civile ne dispose dans les régions susceptibles d'incendies que d'un nombre restreint d'hélicoptères légers du type Alouette II ou Alouette III réservés à I'observation et aux liaisons. 
En été les appareils sont répartis de la façon suivante :

- 1 Alouette II et 1 Alouette III à Nice (Alpes-Maritimes),

- 1 Alouette II au Luc (Var),

- 1 Alouette III en Corse.

Compte tenu du nombre d'hectares de forêts et de maquis brûlés chaque année dans le Midi et en Corse et des risques d'incendie encourus tout le long de la saison chaude, ce dispositif apparait comme dérisoire.

Les appareils lourds ou moyens n'ont été utilisés qu'à titre expérimental :

Les H 34 de l'armée de l'Air ont assuré le transport de commandos du feu au cours de l'été 1966 en Provence et en Corse.

Le Mi 6 soviétique en 1967 a effectué en Provence environ 40 h de vol sur le feu (citerne interne de $11 \mathrm{t}$ d'eau), mais l'expérience s'est terminée tragiquement par la perte de l'appareil et de son équipage.

Le Puma de l'Aérospatiale a effectué en 1971, une trentaine d'heures en Provence et en Corse en mission feu avec seau et participa en 1972 à la mise au point du Seau Alkan et en 1973 à la définition d'une nouvelle technique d'emploi de commandos héliportés. Mais depuis 1971 cet appareil n'a participé à aucune mission réelle dans le dispositif de combat du feu de forêt.

A titre indicatif I'Aviation légère de l'armée de Terre (A.L.A.T.) dispose actuellement de 130 appareils de ce type.

A l'étranger l'hélicoptère dans ce domaine est utilisé de façon courante au Canada et aux États-Unis.

En Californie par exemple le "Department of conservation » de la "California division of forestry » a mis au point, dans les années 1960 une technique d'attaque rapide du feu par commando héliporté constitué de 5 hommes spécialisés dans les feux de forêts; cette technique appelée " helitack operations " a donné d'excellents résultats dans le nord de la Californie en évitant que de simples foyers isolés en zones montagneuses ne deviennent des incendies importants difficiles à éteindre.

Le largage d'eau sur le feu, l'épandage de produits chimiques sont des missions que I'hélicoptère assure régulièrement en cas de sinistres dans cette partie du monde.

Il est intéressant de savoir que les autorités responsables américaines font souvent appel à des compagnies privées exploitant des hélicoptères.

L'hélicoptère ne résoud pas tous les problèmes posés par la prévention et l'extinction des incendies mais, il peut grâce à ses caractéristiques propres et à la souplesse de son emploi jouer un rôle important dans la lutte contre les feux de forêts :

- il accroît l'efficacité du dispositif de combat,

- il facilite la tâche des sauveteurs,

- il complète l'action des moyens aériens traditionnels.

L'hélicoptère peut être, dans le monde, une des chances de survie du patrimoine forestier.

Philippe MILLISCHER

Ingénieur des ventes

AÉROSPATIALE Division hélicoptères

13722 MARIGNANE 